\title{
Vehicle Monitoring System Based on Internet of Things (IoT) for Smart Cities
}

\author{
${ }^{* 1}$ Jitendra Zalke, ${ }^{2}$ Shubham C. Anjankar, ${ }^{3}$ Sandeepkumar R. Pandey, ${ }^{4}$ Noopoor Misal, ${ }^{5}$ Parag Jawarkar \\ 1,4,5 Department of Electronics Design Technology, \\ 2,3 Department of Electronics Engineering, \\ Shri Ramdeobaba College of Engineering and Management, Nagpur-440013, Maharashtra, India \\ Email:zalkej@rknec.edu, anjankarsc@rknec.edu, pandeys@rknec.edu,misaln@rknec.edu, jawarkarps@rknec.edu
}

Received: 10th October 2019, Accepted: 31st January 2020, Published: 29th February 2020

\begin{abstract}
With increase in population, the problems related to transportation systems are increasing day by day, such as constrained car parking facilities, traffic congestion, and in particular traffic rules and road safety. The problem of traffic rules and road safety are becoming very critical for human being in existing metro cities and probably in future for smart cities. This paper discusses the implementation of smart vehicles which gather the information like the speed of the vehicle, the number of persons sitting on/in the vehicle, utilization of safety feature by owner/driver, the location of the vehicle, drunk and drive situation, etc. In case any rules are being violated, the information regarding vehicle will be sent to the central database system, from where the e-challan is generated and send to the concerned authority. Further, the implementation of such features avoids the accidents, provides the safe and secure ride to the persons driving the vehicle.
\end{abstract}

Keywords

IoT, ITS, GPS, Wi-Fi, RTAC

\section{Introduction}

The majority of the Metro cities are known to function on two/four wheelers. Approximately 150 million twowheelers and 30 million of four-wheelers including cars, jeeps, and taxis were registered to the Government of India. So, it's statutory to say that to provide a better traffic management system in Indian scenario, these vehicles need to have more number of smart devices which make them tractable. Even though these statistics are restricted to India, they can be used to correlate with other countries having two/four-wheelers on the road. The two/four wheeler are referred to as vehicle onwards and wherever needs used separately. A smart city, in terms of vehicle management, is expected to have full control of the roads, as well as the vehicles, are driven on it. For this to take place in an efficient manner, the road transport authority (RTA) dedicated to that locality must have information regarding all the vehicles.

The proposed work is based on implementation of smart vehicles which gather the information like the speed of the vehicle, the number of persons sitting on/in the vehicle, utilization of safety feature by owner/driver, the location of the vehicle, drunk and drive situation, etc. In case any rules are being violated, the information regarding vehicle will be sent to the central database system, from where the e-challan is generated and send to the concerned authority. Further, the implementation of such features avoids the accidents, provides the safe and secure ride to the persons driving the vehicle. Combining technologies, we aim to reduce the misuse of vehicles and improve the consensus of law-abiding citizens. The proposed system has the involvement of internet, mobile networks, wireless networks and the communication at each node level. Vehicular nodes communicating with infrastructure over the internet will require a secure architecture as driver/user data will be constantly transmitted. Thus, attention should be paid on the integrity, authenticity and the confidentiality of the data sent over the network of IoT. Since IoT is a multilayer system, the security must be provided according to the issues per layer as well as between layers [1].

\section{Related Work}

The metamorphosis of the internet has given rise to revolutionary technologies such as remote monitoring and control with IoT, cloud computing with IoT, context-aware computing etc. These technologies are now a day's are used meticulously in implementation of concept of smart devices in smart cities. Paper [2], discussed a study on deployments of smart sensors with the mentioned technology to analyze, monitor and control the various parameters in different applications like education, healthcare, agriculture, transportation and many more over the past decades. Amongst all these applications, IoT technology has shown a considerable impact on public transportation system. In recent time, with advent of concept of smart cities, researchers have explored the various remote vehicle monitoring and tracking systems like Vehicular Ad-Hoc Network (VANET), Intelligent Transport System (ITS), [3-6] etc. ITS, 
is used in areas where traffic congestion is a major problem and this problem can be solved using technologies like IoT, RFID, GPS, GPRS, etc. to implement intelligent public transport system [7]. However, this has been incorporated in cars, buses, two-wheelers, etc. Continuous researches are going on, which not only involved the traffic problems but also the incorporation of various things associated with traffic like controlling of the traffic signals according to the traffic density, street light control using IoT for Smart Cities [8]. Implementation of a complex traffic controlling system using embedded PCs has been proposed in [9] but is restricted to the reduction of congestion in traffic in smart cities.

Recent research articles show a major research on the security of vehicles and vehicle communication and its implementation for smart cities. Researcher achieved utmost accuracy to detect vehicle location using GPS in the past [10], and this research have also been used for the application of anti-theft vehicle system. The system has recorded the coordinates of the bike in case it was stolen or hacked [11]. The location-based object detection services and security credential management system (SCMS) for V2X are discussed with respect to vehicle security and communication in $[12,13]$. The article [14] discussed the intelligent transportation system with network security mechanism for control of traffic signal for a vehicle which is promptly arriving at its destination, for the case of vehicle accidents alerts and for providing the data related to vehicle like speed and position of vehicle through which the incident happens and for Road side units (RSUs) with traffic signal control to provide the information to certificate authority.

\section{Overall System Architecture}

The proposed work is based on implementation of vehicle monitoring system based on IoT. The figure (1) shows the overall architecture of the proposed implementation. As shown in figure (1), the vehicle two / four wheeler are equipped with various sensors used to get the precious information about loading on vehicle, speed of the vehicle, condition of driver, etc. Various sensors like load cells, pressure sensor, optical sensor, alcohol sensor, etc. The pressure sensor is used to get the information regarding air pressure in the tyre of the vehicle. The optical sensors are used to measure the speed of the vehicle. The load sensors are proposed to get the information regarding how many people's are sitting on / in two / four-wheeler respectively. The alcohol sensor gives the information of whether driver is in normal or drunk condition.

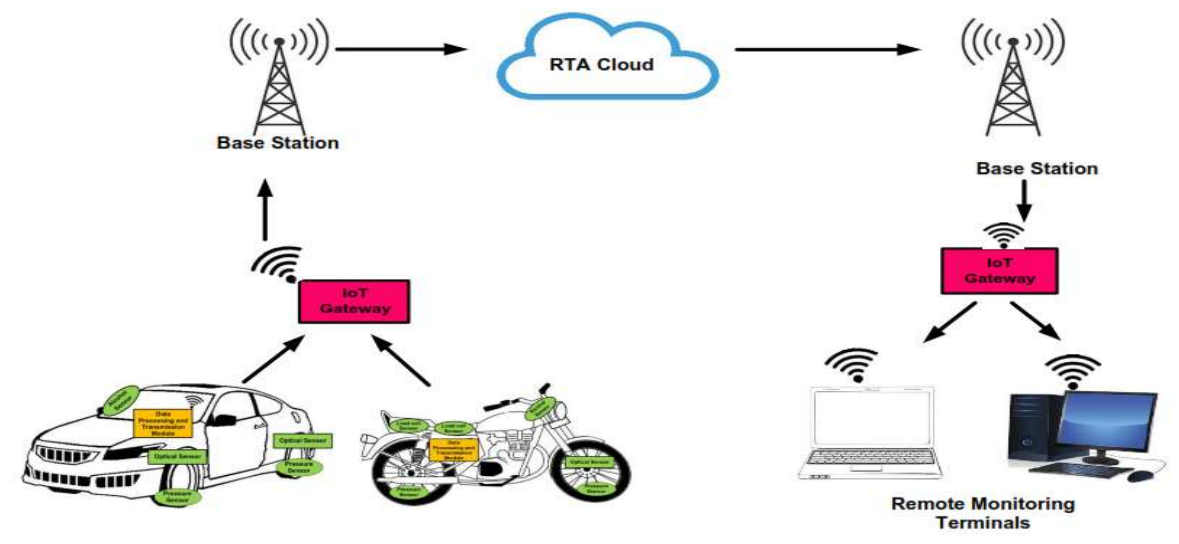

Figure 1: Overall System Implementation

The information generated through sensors will be transmitted by the microcontroller based system. The sensed information will be first stored in memory of the controller installed on the vehicle itself. The controller will establish a connection with the cloud through IoT and upload significant data on it periodically. However, only some of the data will be retained by the cloud, depending upon a predefined algorithm. Once retained, it will be sent to the road transport authority cloud. The remotely placed monitoring system receives the information of vehicle and driver through RTAC. After receiving the data based on predefined algorithm if any threshold violation is there they can generate an e-challan and send it to the driver/owner of the vehicle. The various traffic rules violation taken into consideration for remote monitoring vehicles are: i) Speed of vehicle in particular area and on road. ii) Number of persons sitting on / in two / four wheeler respectively. iii) Condition of driver drunk or normal. 


\section{Methodology}

The Proposed system architecture is as shown in figure (2), consists of processing unit, sensing system mounted on the vehicle as shown in figure (3) and figure (4), locally data display system, and wireless data transmission / receiving module. IoT is the backbone of the complete system implementation.

\section{a) Sensors}

The various sensors like pressure sensor, load sensor, optical sensor and alcohol sensor are mounted on vehicle like two-wheeler vehicle as shown in figure (2) and four-wheeler as shown in figure $(3,4)$. The function of these sensors is to sense the vital parameters, and then send these vital parameters to the remote monitoring station. The sensor system implemented on vehicle has multiple sensors that are used to collect very specific information. The load sensors and pressure sensors are used in this system to get the information regarding how many persons sitting on/in vehicle. The load cells are implemented below the seat. A load cell sensor is used to sense the load of force applied to it and generate a corresponding electrical signal to process the applied load or force. This electrical signal can be processed further to get actual and desired load or force to be applied to anywhere. Considering the average male height in India which is $166.3 \mathrm{~cm}$ and that of women being $152.6 \mathrm{~cm}$, we can estimate that the average weight is 62.2 - $66.7 \mathrm{kgs}$ and 50.8 - $54.4 \mathrm{kgs}$ respectively.

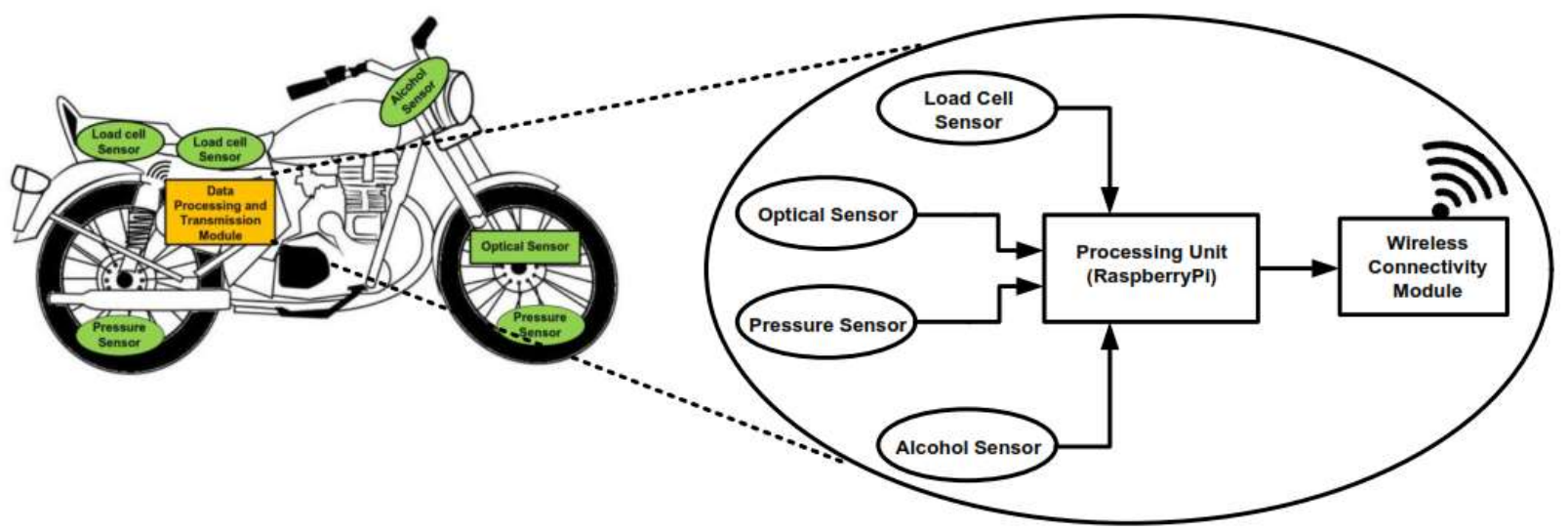

Figure 2: Sensor Implementation on Two-wheeler and Sensor Data Processing

The load sensor senses the load will generate the equivalent electrical signals which will be further given to sophisticated processing unit (Raspberry Pi board). The algorithm in the Raspberry Pi will have a threshold of the average weights of Indian men and women combined. This average weight will simply be multiplied by the processing unit to set the threshold for a certain number of people depending upon the vehicle type (two-wheeler or four-wheeler). The Raspberry Pi will also be aware of the vehicle's ignition. If the weight is beyond the threshold after ignition for more than the specific time set, a penalty in the name of the owner of the vehicle will be generated by the Authority. Also it is possible to detect the number of persons sitting on the vehicle, putting load cell in between two load cell in case of two wheeler as shown in figure(2). Same concept can be extended for four wheeler by placing more sensors and detecting the weight on individual load cell as shown in figure (3) and figure (4).

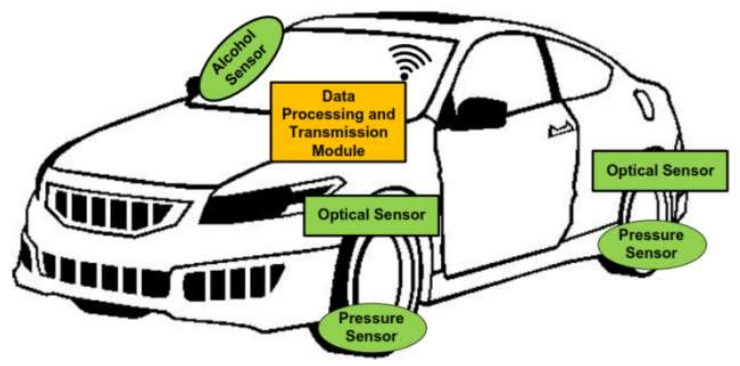

Figure 3: Sensors System Implementation on Four-wheel Vehicle

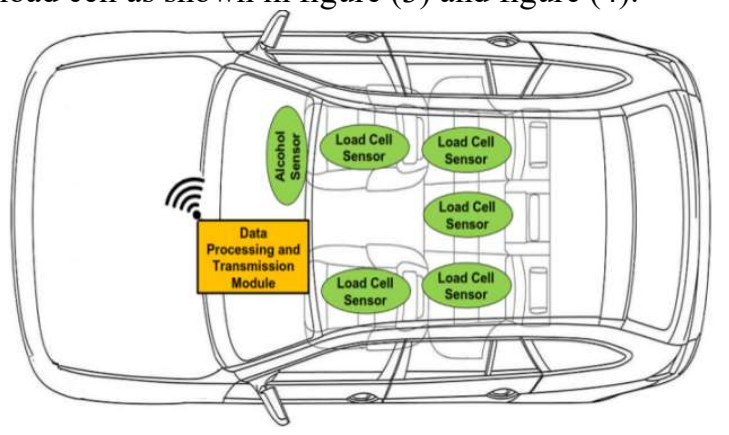

Figure 4: Load Cell Sensors System Implementation on Four-wheel Vehicle 
The optical sensors near the wheels of the vehicle is used to measure a speed of the vehicle when it is moving from certain sensitive area, highway road, critical/danger path etc., where speed limitations are prescribed. For this purpose the location of the vehicle also needs to be monitor simultaneously. Also additional used of the pressure sensors to monitor the air pressure in tyre when moving with certain speed. This is just to alert the driver for safety while driving. The proposed system also uses the alcohol detection sensor to detect whether the driver is intoxicated with a blood alcohol concentration while driving the vehicle. If it founds then also the appropriate immediate action is initiated. Also e-challan will be generated or on site immediate license will also get cancelled.

\section{b) Processing with RaspberryPi:}

The sophisticated processor board of RaspberryPi is used to receive the data from the sensors. The proposed architecture makes the use of RaspberryPi to store the real time speed values and current location sense. The speed will be compared according to the location speed mentioned in server data base. If it exceeds then the information will be sent to the cloud for further action. RaspberryPi is an open-source platform supports IoT and ease of connectivity to the cloud. The proposed board can easily upgraded to newer versions of software without changing the complete hardware system.

\section{c) IoT-Cloud Integration}

A mammoth evolution is seen in IoT and Cloud Computing over the years. As individual IoT can generate the large amount of data from various things and as individual the cloud computing provides a structured pathway for that data to travel to the destination. The integration of both will save lot of money on data centres and allow accessing the data worldwide. The integration of IoT with cloud solves many problems of remote monitoring of the systems like storage of data, remote access to this data by the developers, computing capabilities in terms of virtual machines, etc. Essentially, the Cloud acts as a mediator between things and applications, without the physical use of the complexities necessary for running or working of the application. IoT comprises of a large number of devices or sensors used to sense and generate large amount of non-structured or semi-structured data. This data could be accessed remotely for various purposes like analysis, monitoring, controlling etc. Cloud provides unrestricted, inexpensive, and appealing storage capacity, thus making it the finest and most profitable solution for an arrangement with data generated by IoT. The data stored on the Cloud can be retrieved and processed remotely from anywhere through standard Application Programming Interfaces. The IoT cloud integration offers economical solution for the device connecting, tracking, monitoring and control from anywhere worldwide over the internet.

\section{d) Global Positioning System (GPS)}

GPS is an application of Mobile Wireless Sensor Network in which the mobility of the device plays an important role in an efficient execution. In the proposed work, GPS module is mainly responsible for providing comprehensive information about vehicle location and time. GPS provides the most accurate map showing various sources like towns, cities, etc. The map provides the accurate locations of GPS enabled device. The speciality of GPS is it gives a fast and accurate positioning of the device, irrespective of the poor satellite signal. While tracking the GPS device, it has slowest start time less than 40 seconds, and the fastest start-up time less than 3 seconds, keeping the positioning accuracy to 15 meters.

\section{e) Base Station:}

Every controller on the vehicle will need to send the data logged in a particular to a sub-cloud before it goes to the main Authority cloud. To achieve this, base stations will be installed at certain distances at the road side. If no base stations are present, the provisions are made in the system architecture to access nearby Wi-Fi terminals to transferred the data. Wi-Fi points are now-a-days are available from various sources like malls, shops, industrial offices, etc. Thus, data will be sent to the cloud whenever a Wi-Fi access point is found.

\section{f) Data Communication at Nodes:}

For the purpose of data communication from node to $\mathrm{Wi}-\mathrm{Fi}$, various protocols are used which are operating at the license-free frequency band at frequency of 5130-5835 MHz. This frequency band supports the IEEE 802.11 Wi-Fi standard. This standard is used principally for Wi-Fi access points, which can be extensively used in the cities now-a -days. However, maximum numbers of routers working with this frequency range are used in homes, private businesses, and public gathering places. Specialized routers are also commercially available, which are used in industry and various IoT infrastructures. This has proven to be the most generic way to connect mobile devices such as tablets, laptops and smart phones to the Cloud. Using this, we intend to send the scrutinized data as and when by the controller to the cloud via base stations and also to authority cloud.

\section{Security in System Architecture}

Figure (5), shows the multiple layers in IoT protocol. It consists of four layers viz. sensing, network, service and application layer. The security requirements in each layer are at utmost important. Encryption is the foremost security measure in the system. The encryption is provided at network layer. This will ensure that the information is 
encrypted during the transmission process. This will require the use of plaintext at each node for the encryption and decryption procedures. The application layer has provided the end-to-end security with the help of such encryption and decryption algorithm, so that the information sent and received is restricted to the sender and the receiver. However, since the system deals with personal data of the users, end-to-end encryption is a wise choice as the requirement of privacy is more. The use of key agreement mechanism, Wireless Public Key Infrastructure, security routing, intrusion detection, etc. will be looked into. For communication protocols, the established solutions such as TLS/SSL or IPSec are used. TLS/SSL focuses on the encryption of the link of the transport layer and IPSec focuses on the protection of network layer.

The various cryptographic algorithms are used for the purpose of providing the security such as Advanced Encryption Standard. This algorithm is used to maintain the confidentiality of the data. The Rivest-Shamir-Adelman (RSA) and Elliptic Curve Cryptography are generally used for digital signatures and authentication. The SHA-1 and SHA-256 hash algorithms are used to provide the data integrity.

\begin{tabular}{|c|c|c|}
\hline \multicolumn{3}{|c|}{ Application Layer } \\
\hline \multicolumn{3}{|c|}{ Service Layer } \\
\hline \multicolumn{3}{|c|}{ Network Layer } \\
\hline \multicolumn{3}{|c|}{ Sensing Layer } \\
\hline 4 & 4 & 4 \\
\hline $\begin{array}{c}\text { Information } \\
\text { Security }\end{array}$ & $\begin{array}{l}\text { Physical } \\
\text { Security }\end{array}$ & $\begin{array}{c}\text { Management } \\
\text { Security }\end{array}$ \\
\hline
\end{tabular}

Figure 5: Multiple Layers of IoT.

\section{Result \& Discussion}

The proposed system is tested with a prototype, and it was found that, in case of any deviation to the set parameters for the sensors are being violated, the information regarding vehicle will be sent to the central database system, from where the e-challan is generated and send to the concerned authority. The state-of-the-art IoT and cloud technology is used to implement the proposed system. The load sensor was tested for average weight range of single, double, and triple seat driving, and it was found that, the sensors are sending accurate data for violation of set average weight range. The optical and pressure sensor near the wheels are tested to measure the speed of the vehicle. Also proposed prototype was tested for integration of alcohol detection sensor to detect whether the driver is intoxicated with a blood alcohol concentration while driving the vehicle. It is found that, the appropriate immediate action was initiated and e-challan was generated.

\section{Conclusion}

In this paper, we proposed and discussed the implementation of vehicle monitoring system for smart cities. The proposed implementation is based on IoT and cloud integration to provide the remote monitoring and control of certain rules violated by the user driving the vehicle which otherwise are overlooked. The proposed implementation vehicle sensor network with IoT and cloud integration implement an efficient ITS that increases the safety of road travel and avoids violation of traffic rules. The proposed implementation allows the respective authorities in every country to monitor defiance of rules without any human intervention. The proposed system will improve the monitoring of vehicles.

\section{References}

[1]. Shancang Li, Li Da Xu (2017) Securing the Internet of Things, Syngress, Cambridge.

[2]. Perera C, Zaslavsky A, Christen P, Georgokopoulos D. (2014) Context Aware Computing for The Internet of Things: A Survey. In: Communications Surveys \& Tutorials, vol. 16, pp.414-454, 2014. DOI: 10.1109/SURV.2013.042313.00197

[3]. Huixian Wang, Ren Ping Liu, Wei Ni, Wei Chen, Iain B. Collings, "VANET Modeling and Clustering Design Under Practical Traffic, Channel and Mobility Conditions", IEEE Transactions on Communications, Year: 2015, Volume: 63, Issue: 3, IEEE.

[4]. Dongyao Jia, Kejie Lu, Jianping Wang, “A Disturbance-Adaptive Design for VANET-Enabled Vehicle Platoon”, IEEE Transactions on Vehicular Technology, Year: 2014, Volume: 63, Issue: 2, IEEE. 
[5]. Harry J. F. Qiu, Ivan Wang-Hei Ho, Chi K. Tse, Yu Xie, "A Methodology for Studying 802.11p VANET Broadcasting Performance With Practical Vehicle Distribution", IEEE Transactions on Vehicular Technology, Year: 2015, Volume: 64, Issue: 10. IEEE.

[6] Fernando Terroso-Saenz, Mercedes Valdes-Vela, Cristina Sotomayor-Martinez, Rafael Toledo-Moreo, Antonio F. Gomez-Skarmeta, "A Cooperative Approach to Traffic Congestion Detection With Complex Event Processing and VANET", IEEE Transactions on Intelligent Transportation Systems, June 2012, Volume: 13, Issue: 2.

[7]. Campos L, Cugnasca C, Hirakawa A, Martini J., "Towards an IoT-based System for Smart City", International Symposium on Consumer Electronics (ISCE), pp. 129-130, 2016. DOI: 10.1109/ISCE.2016.7797405

[8]. Ouerhani N, Pazos N, Aeberli M, Muller M., "IoT-Based Dynamic Street Light Control for Smart Cities Use Cases", International Symposium on networks, Computer and Communications (ISNCC), pp.1-5, 2016. DOI: 10.1109/ISNCC.2016.7746112

[9]. Misbahuddin S, Zubairi S, Saggaf A, Basuni J, A-Wadany S, Al-Sofi A., "IoT based Dynamic Road Traffic Management for Smart Cities", 12th International Conference on High-capacity Optical Networks and Enabling/Emerging Technologies (HONET) , pp. 1-5 , 2015. DOI: 10.1109/HONET.2015.7395434

[10]. Koyama Y, Liang T, Tanaka T (2009) High-precision GPS measurement for motorcycle trajectory using kalman filter. In:2009 Sixth International Conference on Networked Sensing Systems (INSS), pp. 1-4, June 2009. DOI: $10.1109 /$ INSS.2009.5409940

[11] Siddharth Kaza, Jennifer Xu, Byron Marshall, Hsinchun Chen, "Topological Analysis of Criminal Activity Networks: Enhancing Transportation Security", IEEE Transactions on Intelligent Transportation Systems, March 2009, Volume: 10, Issue: 1.

[12] Muhammad Usman, Muhammad Rizwan Asghar, Imran Shafique Ansari, Fabrizio Granelli, Khalid A. Qaraqe, "Technologies and Solutions for Location-Based Services in Smart Cities: Past, Present, and Future", IEEE Access April 2018, Volume: 6.

[13] Benedikt Brecht, Dean Therriault, André Weimerskirch, William Whyte, Virendra Kumar, Thorsten Hehn, Roy Goudy, "A Security Credential Management System for V2X Communications", March 2018, Volume: 19, Issue: 12, IEEE.

[14] Hsin-Te Wu, Gwo-Jiun Horng, "Establishing an Intelligent Transportation System With a Network Security Mechanism in an Internet of Vehicle Environment", IEEE Access, September 2017, Volume: 5. 